\title{
Existence of solutions for some implicit partial differential equations and applications to variational integrals involving quasiaffine functions
}

\author{
Bernard DACOROGNA and Ana Margarida RIBEIRO \\ Institut de mathématiques, EPFL, 1015 Lausanne, Switzerland \\ To appear in Proceedings Royal Society of Edinburgh
}

September 13, 2004

\begin{abstract}
We discuss some existence theorems for partial differential inclusions, subject to Dirichlet boundary conditions, of the form

$$
\Phi(D u(x)) \in\{\alpha, \beta\} \text {, a.e. } x \in \Omega,
$$

where $\Phi$ is a quasiaffine function and so, in particular, for $\Phi(D u)=$ $\operatorname{det} D u$.

We then apply it to minimization problems of the form

$$
\inf \left\{\int_{\Omega} g(\Phi(D u(x))) d x: u \in \varphi+W_{0}^{1, \infty}\left(\Omega ; \mathbb{R}^{m}\right)\right\} .
$$
\end{abstract}

\section{Introduction}

In this article we will discuss existence of solutions for some first order partial differential equations and then apply these results to minimization problems of the calculus of variations.

Let us first discuss the model case and introduce some notations (we will always adopt those of [5]). For maps $u: \Omega \subset \mathbb{R}^{n} \longrightarrow \mathbb{R}^{n}$, we will denote its gradient by $D u \in \mathbb{R}^{n \times n}$ and its determinant by $\operatorname{det} D u$.

We will also, given a matrix $\xi \in \mathbb{R}^{n \times n}$, define the singular values of $\xi$ as the eigenvalues of $\left(\xi \xi^{T}\right)^{1 / 2}$ and we will denote them by

$$
0 \leq \lambda_{1}(\xi) \leq \lambda_{2}(\xi) \leq \cdots \leq \lambda_{n}(\xi)
$$

Our first theorem will be 
Theorem 1.1 Let $\Omega \subset \mathbb{R}^{n}$ be a bounded open set, $\alpha<\beta$ and $0<\gamma_{2} \leq \ldots \leq \gamma_{n}$ be such that

$$
\gamma_{2} \prod_{i=2}^{n} \gamma_{i}>\max \{|\alpha|,|\beta|\}
$$

Let $\varphi \in C_{\text {piec }}^{1}\left(\bar{\Omega} ; \mathbb{R}^{n}\right)$ (the set of piecewise $C^{1}$ maps) be such that, for almost every $x \in \Omega$,

$$
\left\{\begin{array}{l}
\alpha<\operatorname{det} D \varphi(x)<\beta, \\
\prod_{i=\nu}^{n} \lambda_{i}(D \varphi(x))<\prod_{i=\nu}^{n} \gamma_{i}, \nu=2, \ldots, n,
\end{array}\right.
$$

then there exists $u \in \varphi+W_{0}^{1, \infty}\left(\Omega ; \mathbb{R}^{n}\right)$ so that

$$
\left\{\begin{array}{l}
\operatorname{det} D u \in\{\alpha, \beta\}, \text { a.e. in } \Omega, \\
\lambda_{\nu}(D u)=\gamma_{\nu}, \nu=2, \ldots, n, \text { a.e. in } \Omega .
\end{array}\right.
$$

Remark 1.2 (i) This theorem generalizes a theorem of Dacorogna-Marcellini [5] where $\beta=-\alpha>0$.

(ii) The theorem is also true if $\alpha=\beta \neq 0$ (the condition $\alpha<\operatorname{det} D \varphi<\beta$, being replaced by $\operatorname{det} D \varphi=\alpha$ ) and therefore also generalizes a theorem of Dacorogna-Tanteri [9].

We then apply this theorem (for details see Theorem 5.1) to the following minimization problem

$$
(P) \quad \inf \left\{\int_{\Omega} g(\operatorname{det} D u(x)) d x: u \in \varphi+W_{0}^{1, \infty}\left(\Omega ; \mathbb{R}^{n}\right)\right\} .
$$

This problem is important for applications (see [2] and [3]).

It should immediately be pointed out that even when $g$ is convex, it is not clear that $(P)$ admits a minimizer (unless $\varphi$ is affine and in which case $u=\varphi$ is a minimizer). It was proved in [2] and then extended in [6], that if $\Omega$ is smooth and $\varphi$ is a $C^{1, \alpha}, 0<\alpha<1$, diffeomorphism then there exists a minimizer $\bar{u}$ of $(P)$, that also solves

$$
\left\{\begin{array}{l}
\operatorname{det} D \bar{u}=\frac{1}{|\Omega|} \int_{\Omega} \operatorname{det} D \varphi(y) d y, \text { in } \Omega, \\
\bar{u}=\varphi, \text { on } \partial \Omega .
\end{array}\right.
$$

The non convex case was then investigated by Mascolo-Schianchi [10] for non affine $\varphi$ and by Cellina-Zagatti [1] and Dacorogna-Marcellini [4] when $\varphi$ is affine. Theorem 1.1 will allow us to give a new proof of the existence of minimizers for $(P)$ when $g$ is non convex.

We then discuss the case of quasiaffine functions. We recall that for $m=$ $n=2$ (for the general case, $m, n \geq 2$, see Section 2) a quasiaffine function is of the form

$$
\Phi(\xi)=\Phi(0)+<\mu_{1} ; \xi>+\mu_{2} \operatorname{det} \xi,
$$


where $\mu_{1} \in \mathbb{R}^{2 \times 2}$ and $\mu_{2} \in \mathbb{R}$.

We will then prove the following theorem, which is from some aspects more general than Theorem 1.1 (since we can allow general quasiaffine functions) and from some other parts weaker (since we cannot prescribe other equations such as $\lambda_{i}(D u)=\gamma_{i}$; for some extensions see [11]).

Theorem 1.3 Let $\Omega \subset \mathbb{R}^{n}$ be a bounded open set, $\alpha<\beta, \Phi: \mathbb{R}^{m \times n} \longrightarrow \mathbb{R} a$ non constant quasiaffine function and $\varphi \in C_{\text {piec }}^{1}\left(\bar{\Omega} ; \mathbb{R}^{m}\right)$ such that, for almost every $x \in \Omega$,

$$
\alpha<\Phi(D \varphi(x))<\beta \text {. }
$$

Then there exists $u \in \varphi+W_{0}^{1, \infty}\left(\Omega ; \mathbb{R}^{m}\right)$ satisfying

$$
\Phi(D u) \in\{\alpha, \beta\} \text {, a.e. in } \Omega \text {. }
$$

This theorem has a direct application to the minimization problem

$$
\inf \left\{\int_{\Omega} g(\Phi(D u(x))) d x: u \in \varphi+W_{0}^{1, \infty}\left(\Omega ; \mathbb{R}^{m}\right)\right\}
$$

when $g$ is non convex, recovering a theorem already proved, by different means, by Cellina-Zagatti [1].

\section{Preliminaries}

In this preliminaries we will state the main abstract existence theorem that we will use in the following sections and we will also briefly define the notion of quasiaffine function.

We start by recalling the notion of rank one convex hull of a given set (for more details, see [5]).

Notation 2.1 We let, for $E \subset \mathbb{R}^{m \times n}$,

$$
\overline{\mathcal{F}}_{E}=\left\{f: \mathbb{R}^{m \times n} \rightarrow \overline{\mathbb{R}}=\mathbb{R} \cup\{+\infty\} \text { and }\left.f\right|_{E} \leq 0\right\}
$$

Rco $E=\left\{\xi \in \mathbb{R}^{m \times n}: f(\xi) \leq 0\right.$, for every rank one convex $\left.f \in \overline{\mathcal{F}}_{E}\right\}$.

We will denote by int Rco $E$ the interior of the rank one convex hull of $E$.

We start with the following definition introduced by Dacorogna-Marcellini in [5], which is the key condition to get existence of solutions.

Definition 2.2 (Approximation property) Let $E \subset K(E) \subset \mathbb{R}^{m \times n}$. The sets $E$ and $K(E)$ are said to have the approximation property if there exists a family of closed sets $E_{\delta}$ and $K\left(E_{\delta}\right), \delta>0$, such that

(1) $E_{\delta} \subset K\left(E_{\delta}\right) \subset$ int $K(E)$ for every $\delta>0$;

(2) for every $\varepsilon>0$ there exists $\delta_{0}=\delta_{0}(\varepsilon)>0$ such that $\operatorname{dist}(\eta ; E) \leq \varepsilon$ for every $\eta \in E_{\delta}$ and $\delta \in\left[0, \delta_{0}\right]$;

(3) if $\eta \in \operatorname{int} K(E)$ then $\eta \in K\left(E_{\delta}\right)$ for every $\delta>0$ sufficiently small. 
The main abstract existence theorem that we will use in our analysis is (cf. Theorem 6.3 combined with Theorem 6.14 in [5], or for a slightly more general version see Dacorogna-Pisante [7]).

Theorem 2.3 Let $\Omega \subset \mathbb{R}^{n}$ be open. Let $E \subset \mathbb{R}^{m \times n}$ be compact. Assume that Rco $E$ has the approximation property with $K\left(E_{\delta}\right)=\operatorname{Rco} E_{\delta}$. Let $\varphi \in$ $C_{\text {piec }}^{1}\left(\bar{\Omega} ; \mathbb{R}^{m}\right)$ (where $C_{\text {piec }}^{1}$ denotes the set of piecewise $C^{1}$ maps) be such that

$$
D \varphi(x) \in E \cup \operatorname{int} \operatorname{Rco} E \text {, a.e. in } \Omega \text {. }
$$

Then there exists (a dense set of) $u \in \varphi+W_{0}^{1, \infty}\left(\Omega ; \mathbb{R}^{m}\right)$ such that

$$
D u(x) \in E \text {, a.e. in } \Omega \text {. }
$$

Finally we recall the notion of quasiaffine functions, cf. for more details [3].

Definition 2.4 We say that $\Phi: \mathbb{R}^{m \times n} \rightarrow \mathbb{R}$ is quasiaffine if

$$
\Phi(\xi)=\Phi(0)+\sum_{k=1}^{m \wedge n}<A^{k} ; \operatorname{adj}_{k} \xi>,
$$

where $m \wedge n=\min \{n, m\}, A^{k} \in \mathbb{R}^{\sigma(k)}, \sigma(k)=\left(\begin{array}{c}m \\ k\end{array}\right) \times\left(\begin{array}{c}n \\ k\end{array}\right), \operatorname{adj}_{k} \xi$ is the matrix of the minors of $\xi$ of order $k$ and $\langle;>$ denotes the scalar product.

In an equivalent form, we can write

$$
\Phi(\xi)=\Phi(0)+\sum_{q=1}^{m \wedge n} \sum_{\substack{1 \leq i_{1}<\cdots<i_{q} \leq m \\
1 \leq j_{1}<\cdots<j_{q} \leq n}} \mu_{j_{1} \cdots j_{q}}^{i_{1} \cdots i_{q}} \operatorname{det}\left(\begin{array}{ccc}
\xi_{j_{1}}^{i_{1}} & \cdots & \xi_{j_{q}}^{i_{1}} \\
\vdots & & \vdots \\
\xi_{j_{1}}^{i_{q}} & \cdots & \xi_{j_{q}}^{i_{q}}
\end{array}\right),
$$

for some constants $\mu_{j_{1} \cdots j_{q}}^{i_{1} \cdots i_{q}} \in \mathbb{R}, 1 \leq q \leq m \wedge n$.

We moreover have

Proposition 2.5 Let $\Phi: \mathbb{R}^{m \times n} \rightarrow \mathbb{R}$ be quasiaffine and $\Omega \subset \mathbb{R}^{n}$ be a bounded open set. Then

$$
\int_{\Omega} \Phi(D v(x)) d x=\int_{\Omega} \Phi(D u(x)) d x, \forall v \in u+W_{0}^{1, \infty}\left(\Omega ; \mathbb{R}^{m}\right) .
$$

\section{Rank one convex hulls}

In this section we will compute the rank one convex hull of sets $E$ involving the condition

$$
\Phi(\xi) \in\{\alpha, \beta\},
$$

where $\Phi$ is a quasiaffine function. We start in Section 3.1 by the case of the determinant where extra conditions on the singular values are allowed. In Section 3.2 we will deal with general quasiaffine functions. 


\subsection{The case of the determinant}

The theorem we will prove is the following.

Theorem 3.1 Let $\alpha \leq \beta, 0<\gamma_{2} \leq \ldots \leq \gamma_{n}$ be constants so that

$$
\gamma_{2} \prod_{i=2}^{n} \gamma_{i} \geq \max \{|\alpha|,|\beta|\}
$$

Let

$$
E=\left\{\xi \in \mathbb{R}^{n \times n}: \operatorname{det} \xi \in\{\alpha, \beta\}, \lambda_{i}(\xi)=\gamma_{i}, i=2, \ldots, n\right\}
$$

then

$$
\operatorname{Rco} E=\left\{\xi \in \mathbb{R}^{n \times n}: \operatorname{det} \xi \in[\alpha, \beta], \prod_{i=\nu}^{n} \lambda_{i}(\xi) \leq \prod_{i=\nu}^{n} \gamma_{i}, \nu=2, \ldots, n\right\} .
$$

Moreover, if $\alpha<\beta$,

$$
\operatorname{int} \operatorname{Rco} E=\left\{\xi \in \mathbb{R}^{n \times n}: \operatorname{det} \xi \in(\alpha, \beta), \prod_{i=\nu}^{n} \lambda_{i}(\xi)<\prod_{i=\nu}^{n} \gamma_{i}, \nu=2, \ldots, n\right\}
$$

and if $\alpha=\beta$

$$
\text { int Rco } E=\left\{\xi \in \mathbb{R}^{n \times n}: \operatorname{det} \xi=\alpha, \prod_{i=\nu}^{n} \lambda_{i}(\xi)<\prod_{i=\nu}^{n} \gamma_{i}, \nu=2, \ldots, n\right\}
$$

where the interior is to be understood relative to the manifold $\{\operatorname{det} \xi=\alpha\}$.

Remark 3.2 The theorem extends [8] and [5] if $\beta=-\alpha>0$ and [9] if $\alpha=\beta$. In particular note that if we let, when $\beta=-\alpha>0$,

$$
\gamma_{1}=\beta\left(\prod_{i=2}^{n} \gamma_{i}\right)^{-1}
$$

then

$$
\begin{aligned}
E & =\left\{\xi \in \mathbb{R}^{n \times n}: \operatorname{det} \xi \in\{-\beta, \beta\}, \lambda_{i}(\xi)=\gamma_{i}, i=2, \ldots, n\right\} \\
& =\left\{\xi \in \mathbb{R}^{n \times n}: \lambda_{1}(\xi)=\gamma_{1}, \lambda_{i}(\xi)=\gamma_{i}, i=2, \ldots, n\right\}
\end{aligned}
$$

Proof. We will divide the proof into two parts. In the first one we will obtain the characterization of Rco $E$ and in the second a characterization of its interior. Part 1. We let

$$
X=\left\{\xi \in \mathbb{R}^{n \times n}: \operatorname{det} \xi \in[\alpha, \beta], \prod_{i=\nu}^{n} \lambda_{i}(\xi) \leq \prod_{i=\nu}^{n} \gamma_{i}, \nu=2, \ldots, n\right\}
$$

and we wish to show that $X=\operatorname{Rco} E$. 
Step 1: Rco $E \subset X$. This is the easy implication. Indeed observe that $E \subset X$ and that the functions

$$
\xi \rightarrow \pm \operatorname{det} \xi, \quad \xi \rightarrow \prod_{i=\nu}^{n} \lambda_{i}(\xi), \nu=2, \ldots, n
$$

are rank one convex (see [5]). We therefore have that the set $X$ is rank one convex and thus the desired inclusion.

Step 2: $X \subset$ Rco $E$. Since the set $X$ is compact (the function $\xi \rightarrow \lambda_{n}(\xi)$ being a norm), it is enough to show that $\partial X \subset$ Rco $E$. So we let $\xi \in \partial X$ and we wish to prove that $\xi \in \operatorname{Rco} E$. Note that $\partial X=X_{\alpha} \cup X_{\beta} \cup X_{2} \cup \cdots \cup X_{n}$ where

$$
\begin{gathered}
X_{\alpha}=\{\xi \in X: \operatorname{det} \xi=\alpha\} \quad X_{\beta}=\{\xi \in X: \operatorname{det} \xi=\beta\} \\
X_{\nu}=\left\{\xi \in X: \prod_{i=\nu}^{n} \lambda_{i}(\xi)=\prod_{i=\nu}^{n} \gamma_{i}\right\}, \quad \nu=2, \ldots, n .
\end{gathered}
$$

Since all the functions involved in the definition of $X$ are right and left $S O(n)$ invariant, there is no loss of generality in assuming that $\xi$ is diagonal

$$
\xi=\operatorname{diag}\left(x_{1}, x_{2}, \ldots, x_{n}\right)
$$

with $0 \leq\left|x_{1}\right| \leq x_{2} \leq \cdots \leq x_{n}$. We therefore have $\lambda_{1}(\xi)=\left|x_{1}\right|, \lambda_{i}(\xi)=x_{i}, i=$ $2, \ldots, n$. We will now proceed by induction on the dimension $n$; when $n=1$ the result is trivial.

Several possibilities can then happen, bearing in mind that $\xi \in \partial X$.

Case 1: $\xi \in X_{\bar{\nu}}$ for a certain $\bar{\nu}=2, \ldots, n$ (i.e. $\prod_{i=\bar{\nu}}^{n} x_{i}=\prod_{i=\bar{\nu}}^{n} \gamma_{i}$ ). We write $\xi \in \mathbb{R}^{n \times n}$ as two blocks, one in $\mathbb{R}^{(\bar{\nu}-1) \times(\bar{\nu}-1)}$ and one in $\mathbb{R}^{(n-\bar{\nu}+1) \times(n-\bar{\nu}+1)}$ in the following way $\xi=\operatorname{diag}\left(\xi_{\bar{\nu}-1}, \xi_{n-\bar{\nu}+1}\right)$ where $\xi_{\bar{\nu}-1}=\operatorname{diag}\left(x_{1}, \ldots, x_{\bar{\nu}-1}\right)$ and $\xi_{n-\bar{\nu}+1}=\operatorname{diag}\left(x_{\bar{\nu}}, \ldots, x_{n}\right)$.

We then apply the hypothesis of induction on $\xi_{\bar{\nu}-1}$ and $\xi_{n-\bar{\nu}+1}$ (we will check that we can do so below) and we deduce that $\xi \in$ Rco $E$. Let us now see that we can apply the hypothesis of induction first for $\xi_{\bar{\nu}-1}$. We have (when $\bar{\nu}=2$ or $\bar{\nu}=n$, terms such as $\prod_{i=2}^{\bar{\nu}-1}$ or $\prod_{i=\bar{\nu}+1}^{n}$ should be replaced by 1 )

$$
\begin{gathered}
\gamma_{2} \prod_{i=2}^{\bar{\nu}-1} \gamma_{i}=\gamma_{2} \prod_{i=2}^{n} \gamma_{i}\left(\prod_{i=\bar{\nu}}^{n} \gamma_{i}\right)^{-1} \geq \max \left\{\frac{|\alpha|}{\gamma_{\bar{\nu}} \cdots \gamma_{n}}, \frac{|\beta|}{\gamma_{\bar{\nu}} \cdots \gamma_{n}}\right\} \\
\operatorname{det} \xi_{\bar{\nu}-1}=\prod_{i=1}^{\bar{\nu}-1} x_{i}=\prod_{i=1}^{n} x_{i}\left(\prod_{i=\bar{\nu}}^{n} x_{i}\right)^{-1}=\prod_{i=1}^{n} x_{i}\left(\prod_{i=\bar{\nu}}^{n} \gamma_{i}\right)^{-1} \\
=\operatorname{det} \xi\left(\prod_{i=\bar{\nu}}^{n} \gamma_{i}\right)^{-1} \in\left[\frac{\alpha}{\gamma_{\bar{\nu}} \cdots \gamma_{n}}, \frac{\beta}{\gamma_{\bar{\nu}} \cdots \gamma_{n}}\right]
\end{gathered}
$$




$$
\prod_{i=\nu}^{\bar{\nu}-1} \lambda_{i}\left(\xi_{\bar{\nu}-1}\right)=\prod_{i=\nu}^{n} x_{i}\left(\prod_{i=\bar{\nu}}^{n} x_{i}\right)^{-1}=\prod_{i=\nu}^{n} x_{i}\left(\prod_{i=\bar{\nu}}^{n} \gamma_{i}\right)^{-1} \leq \prod_{i=\nu}^{\bar{\nu}-1} \gamma_{i}, \nu=2, \ldots, \bar{\nu}-1
$$

and thus the result.

Similarly for $\xi_{n-\bar{\nu}+1}$ since (here the role of $\alpha$ and $\beta$ is played, for both, by $\left.\prod_{i=\bar{\nu}}^{n} \gamma_{i}\right)$

$$
\begin{gathered}
\gamma_{\bar{\nu}+1} \prod_{i=\bar{\nu}+1}^{n} \gamma_{i} \geq \prod_{i=\bar{\nu}}^{n} \gamma_{i}, \\
\operatorname{det} \xi_{n-\bar{\nu}+1}=\prod_{i=\bar{\nu}}^{n} x_{i}=\prod_{i=\bar{\nu}}^{n} \gamma_{i}, \\
\prod_{i=\nu-\bar{\nu}+1}^{n-\bar{\nu}+1} \lambda_{i}\left(\xi_{n-\bar{\nu}+1}\right)=\prod_{i=\nu}^{n} x_{i} \leq \prod_{i=\nu}^{n} \gamma_{i}, \nu=\bar{\nu}+1, \ldots, n
\end{gathered}
$$

we have the claim.

Case 2: $\xi \in X_{\alpha}$ (similarly for the case $\xi \in X_{\beta}$ ). We can also assume that $\xi \notin X_{\nu}, \nu=2, \ldots, n$, otherwise we apply Case 1 . So we can assume that

$$
\xi \in \operatorname{int} X_{\alpha}=\left\{\eta \in \mathbb{R}^{n \times n}: \operatorname{det} \eta=\alpha, \prod_{i=\nu}^{n} \lambda_{i}(\eta)<\prod_{i=\nu}^{n} \gamma_{i}, \nu=2, \ldots, n\right\} .
$$

This is clearly an open set (relative to the manifold $\{\operatorname{det} \eta=\alpha\}$ ).

Recall that

$$
\xi=\operatorname{diag}\left(x_{1}, \ldots, x_{n}\right)=\left(\begin{array}{ccc}
x_{1} & & \\
& \ddots & \\
& & x_{n}
\end{array}\right) .
$$

We then set for $t \in \mathbb{R}$

$$
\xi_{t}=\left(\begin{array}{cccc}
x_{1} & & & \\
& \ddots & & \\
& & x_{n-1} & t \\
& & 0 & x_{n}
\end{array}\right)
$$

and observe that $\operatorname{det} \xi_{t}=\operatorname{det} \xi=\alpha$. Since int $X_{\alpha}$ is bounded we can find $t_{1}<0<t_{2}$ so that $\xi_{t_{1}}, \xi_{t_{2}} \in \partial X_{\alpha}$ which means that $\xi_{t_{i}} \in X_{\nu_{i}}, i=1,2$, for a certain $\nu_{i}=2, \ldots, n$ and therefore, by Case $1, \xi_{t_{i}} \in$ Rco $E$ and thus, since $\operatorname{rank}\left(\xi_{t_{1}}-\xi_{t_{2}}\right)=1$, we deduce that $\xi \in$ Rco $E$ as wished.

This concludes the first part of the theorem.

Part 2. The representation formula for int Rco $E$ is easy and its proof is very similar to the ones in [5] or [8] and we skip the details. 


\subsection{The case of a quasiaffine function}

We will need, prior to the main theorem, two elementary lemmas but we postpone their proofs to the end of the present subsection. The first one will be used to assert that condition (3.1) below can be fulfilled by some $c_{j}^{i}>0$ and will also be used in Theorem 1.3. Lemma 3.4 will be used in the proof of Theorem 3.5.

Lemma 3.3 Let $\Phi: \mathbb{R}^{m \times n} \longrightarrow \mathbb{R}$ be a non constant quasiaffine function and $M, N>0$. Then there exist $c_{j}^{i}>N, i=1, \ldots, m, j=1, \ldots, n$ so that

$$
\inf \left\{|\Phi(\xi)|:\left|\xi_{j}^{i}\right|=c_{j}^{i}\right\}>M
$$

Lemma 3.4 Let $\Phi: \mathbb{R}^{m \times n} \longrightarrow \mathbb{R}$ be a non constant quasiaffine function. Then $\Phi$ has no local extremum.

We can now state the main theorem.

Theorem 3.5 Let $\Phi: \mathbb{R}^{m \times n} \longrightarrow \mathbb{R}$ be a non constant quasiaffine function, $\alpha<\beta, c_{j}^{i}>0$ satisfying

$$
\inf \left\{|\Phi(\xi)|:\left|\xi_{j}^{i}\right|=c_{j}^{i}\right\}>\max \{|\alpha|,|\beta|\} .
$$

Let

$$
E=\left\{\xi \in \mathbb{R}^{m \times n}: \Phi(\xi) \in\{\alpha, \beta\},\left|\xi_{j}^{i}\right| \leq c_{j}^{i}, i=1, \ldots, m, j=1, \ldots, n\right\}
$$

then

$$
\operatorname{Rco} E=\left\{\xi \in \mathbb{R}^{m \times n}: \Phi(\xi) \in[\alpha, \beta],\left|\xi_{j}^{i}\right| \leq c_{j}^{i}, i=1, \ldots, m, j=1, \ldots, n\right\},
$$

int Rco $E=\left\{\xi \in \mathbb{R}^{m \times n}: \Phi(\xi) \in(\alpha, \beta),\left|\xi_{j}^{i}\right|<c_{j}^{i}, i=1, \ldots, m, j=1, \ldots, n\right\}$.

Proof. Part 1. We let

$$
X=\left\{\xi \in \mathbb{R}^{m \times n}: \Phi(\xi) \in[\alpha, \beta],\left|\xi_{j}^{i}\right| \leq c_{j}^{i}, i=1, \ldots, m, j=1, \ldots, n\right\}
$$

and we show that $X=\operatorname{Rco} E$. The inclusion Rco $E \subset X$ follows from the combination of the facts that $E \subset X$ and that the set $X$ is rank one convex (the functions $\Phi,-\Phi$ and $|\cdot|$ being rank one convex).

We therefore have to show only that $X \subset \operatorname{Rco} E$. So we let $\xi \in X$ and we can assume that $\alpha<\Phi(\xi)<\beta$ otherwise the result is trivial. We observe that (3.1) implies that for every $\xi \in X$ there exists $(i, j)$ so that $\left|\xi_{j}^{i}\right|<c_{j}^{i}$. So let for $t \in \mathbb{R}$

$$
\xi^{t}=\xi+t e^{i} \otimes e_{j}
$$

and observe that by compactness there exist $t_{1}<0<t_{2}$ so that $\xi^{t_{\nu}} \in \partial X$, $\nu=1,2$ which implies that either $\Phi\left(\xi^{t_{\nu}}\right) \in\{\alpha, \beta\}$ or $\left|\left(\xi^{t_{\nu}}\right)_{j}^{i}\right|=c_{j}^{i}, \nu=1,2$. If the first possibility happens then we are done, if however the second case holds then we restart the process with a different $(i, j)$, since it is not possible by $(3.1)$ that $\left|\left(\xi^{t_{\nu}}\right)_{j}^{i}\right|=c_{j}^{i}$ for every $(i, j)$. 
Part 2. We now define

$$
Y=\left\{\xi \in \mathbb{R}^{m \times n}: \Phi(\xi) \in(\alpha, \beta),\left|\xi_{j}^{i}\right|<c_{j}^{i}, i=1, \ldots, m, j=1, \ldots, n\right\}
$$

and observe that since $Y \subset$ Rco $E$ and $Y$ is open, then $Y \subset$ int Rco $E$. So let us show the reverse inclusion and choose $\xi \in \operatorname{int}$ Rco $E$. Clearly such a $\xi$ must have $\left|\xi_{j}^{i}\right|<c_{j}^{i}$. Lemma 3.4 shows also that $\xi$ should be so that $\alpha<\Phi(\xi)<\beta$. These observations imply the result.

We now prove Lemma 3.3.

Proof. Since $\Phi$ is quasiaffine, we can write

$$
\Phi(\xi)=\Phi(0)+\sum_{q=1}^{m \wedge n} \sum_{\substack{1 \leq i_{1}<\cdots<i_{q} \leq m \\
1 \leq j_{1}<\cdots<j_{q} \leq n}} \mu_{j_{1} \cdots j_{q}}^{i_{1} \cdots i_{q}} \operatorname{det}\left(\begin{array}{ccc}
\xi_{j_{1}}^{i_{1}} & \cdots & \xi_{j_{q}}^{i_{1}} \\
\vdots & & \vdots \\
\xi_{j_{1}}^{i_{q}} & \cdots & \xi_{j_{q}}^{i_{q}}
\end{array}\right) .
$$

Since $\Phi$ is not constant we can find $1 \leq s \leq m \wedge n, 1 \leq i_{1}<\cdots<i_{s} \leq m$ and $1 \leq j_{1}<\cdots<j_{s} \leq n$ so that $\mu_{j_{1} \cdots j_{s}}^{i_{1} \cdots i_{s}} \neq 0$ and $\mu_{j_{1} \cdots j_{q}}^{i_{1} \cdots i_{q}}=0, \forall q>s$. Assume without loss of generality that

$$
\mu_{1 \cdots s}^{1 \cdots s} \neq 0 .
$$

Let us define the set

$$
\Theta=\left\{\theta \in \mathbb{R}^{m \times n}: \theta_{j}^{i} \in\{ \pm 1\}\right\}
$$

and the product $A \odot B \in \mathbb{R}^{m \times n}$, for two given matrixes $A, B \in \mathbb{R}^{m \times n}$, as

$$
(A \odot B)_{j}^{i}=A_{j}^{i} \cdot B_{j}^{i} .
$$

We want to find a matrix $C \in \mathbb{R}^{m \times n}$ such that $c_{j}^{i}>N$ and

$$
\xi=C \odot \theta, \theta \in \Theta \Longrightarrow|\Phi(\xi)|>M .
$$

In fact we will prove that the matrix can be chosen of the form $C=\tau A$ where $\tau>0$ and for $t>0$

$$
\begin{aligned}
& A_{i}^{i}=t \text { if } 1 \leq i \leq s, \\
& A_{j}^{i}=1 \text { if not (i.e. if } i \neq j \text { or if } i=j \geq s+1 \text { ). }
\end{aligned}
$$

We observe that

$$
\begin{aligned}
\Phi(\xi) & =\Phi(C \odot \theta) \\
& =\Phi(0)+\sum_{q=1}^{s} \tau^{q} \sum_{\substack{1 \leq i_{1}<\cdots<i_{q} \leq m \\
1 \leq j_{1}<\cdots<j_{q} \leq n}} \mu_{j_{1} \cdots j_{q}}^{i_{1} \cdots i_{q}} \operatorname{det}\left(\begin{array}{cccc}
A_{j_{1}}^{i_{1}} \theta_{j_{1}}^{i_{1}} & \cdots & A_{j_{q}}^{i_{1}} \theta_{j_{q}}^{i_{1}} \\
\vdots & & \vdots \\
A_{j_{1}}^{i_{q}} \theta_{j_{1}}^{i_{q}} & \cdots & A_{j_{q}}^{i_{q}} \theta_{j_{q}}^{i_{q}}
\end{array}\right)
\end{aligned}
$$


and that for $\tau$ and $t$ sufficiently large it is possible to find $\gamma>0$ so that

$$
|\Phi(\xi)| \geq \gamma \tau^{s} t^{s} .
$$

So choosing $\tau$ and $t$ sufficiently large we have indeed found $c_{j}^{i}>N$ and $|\Phi(\xi)|>$ $M$ as wished.

We now prove Lemma 3.4.

Proof. We will prove that if $\Phi$ has a local extremum then it must be constant. We proceed in two steps.

Step 1: We first show that if $\xi$ is a local extremum point of $\Phi$, then $\Phi$ is constant in a neighborhood of $\xi$.

Assume that $\xi$ is a local minimum point of $\Phi$ (the case of a local maximizer being handled similarly). We therefore have that there exists $\varepsilon>0$ so that

$$
\Phi(\xi) \leq \Phi(\xi+v) \text {, for every } v \in \mathbb{R}^{m \times n} \text { so that }\left|v_{j}^{i}\right| \leq \varepsilon .
$$

Let us show that this implies that

$$
\Phi(\xi)=\Phi(\xi+v) \text {, for every } v \in \mathbb{R}^{m \times n} \text { so that }\left|v_{j}^{i}\right| \leq \varepsilon .
$$

We write

$$
v=\sum_{\substack{1 \leq i \leq m \\ 1 \leq j \leq n}} v_{j}^{i} e^{i} \otimes e_{j}
$$

and observe that, since $\Phi$ is quasiaffine,

$$
\Phi(\xi)=\frac{1}{2} \Phi\left(\xi+v_{1}^{1} e^{1} \otimes e_{1}\right)+\frac{1}{2} \Phi\left(\xi-v_{1}^{1} e^{1} \otimes e_{1}\right)
$$

and since (3.3) is satisfied we deduce that

$$
\Phi\left(\xi \pm v_{1}^{1} e^{1} \otimes e_{1}\right)=\Phi(\xi),\left|v_{1}^{1}\right| \leq \varepsilon .
$$

We next write, using again the fact that $\Phi$ is quasiaffine,

$\Phi\left(\xi+v_{1}^{1} e^{1} \otimes e_{1}\right)=\frac{1}{2} \Phi\left(\xi+v_{1}^{1} e^{1} \otimes e_{1}+v_{2}^{1} e^{1} \otimes e_{2}\right)+\frac{1}{2} \Phi\left(\xi+v_{1}^{1} e^{1} \otimes e_{1}-v_{2}^{1} e^{1} \otimes e_{2}\right)$

and since (3.3) and (3.5) hold, we deduce that

$$
\Phi\left(\xi+v_{1}^{1} e^{1} \otimes e_{1} \pm v_{2}^{1} e^{1} \otimes e_{2}\right)=\Phi\left(\xi+v_{1}^{1} e^{1} \otimes e_{1}\right)=\Phi(\xi),\left|v_{1}^{1}\right|,\left|v_{2}^{1}\right| \leq \varepsilon .
$$

Iterating the procedure we have indeed established (3.4).

Step 2: We now show that if $\Phi$ is locally constant around a point $\xi \in \mathbb{R}^{m \times n}$ then $\Phi$ is constant everywhere establishing the result. So assume that

$$
\Phi(\xi+v)=\Phi(\xi), \forall v \in \mathbb{R}^{m \times n} \text { with }\left|v_{j}^{i}\right| \leq \varepsilon
$$


and let us show that

$$
\Phi(\xi+w)=\Phi(\xi), \forall w \in \mathbb{R}^{m \times n} .
$$

The procedure is similar to that of Step 1 and we start to show that for all $w_{1}^{1} \in \mathbb{R}$ and $\left|v_{j}^{i}\right| \leq \varepsilon$ we have

$$
\Phi\left(\xi+w_{1}^{1} e^{1} \otimes e_{1}+\sum_{(i, j) \neq(1,1)} v_{j}^{i} e^{i} \otimes e_{j}\right)=\Phi\left(\xi+w_{1}^{1} e^{1} \otimes e_{1}\right)=\Phi(\xi) .
$$

Indeed if $\left|w_{1}^{1}\right| \leq \varepsilon$ this is nothing else than (3.6) so we may assume that $\left|w_{1}^{1}\right|>\varepsilon$ and use the fact that $\Phi$ is quasiaffine, to deduce that

$$
\begin{aligned}
\Phi(\xi & \left.+\varepsilon \frac{w_{1}^{1}}{\left|w_{1}^{1}\right|} e^{1} \otimes e_{1}+\sum_{(i, j) \neq(1,1)} v_{j}^{i} e^{i} \otimes e_{j}\right)= \\
& =\frac{\varepsilon}{\left|w_{1}^{1}\right|} \Phi\left(\xi+w_{1}^{1} e^{1} \otimes e_{1}+\sum_{(i, j) \neq(1,1)} v_{j}^{i} e^{i} \otimes e_{j}\right)+ \\
& +\left(1-\frac{\varepsilon}{\left|w_{1}^{1}\right|}\right) \Phi\left(\xi+\sum_{(i, j) \neq(1,1)} v_{j}^{i} e^{i} \otimes e_{j}\right) .
\end{aligned}
$$

Therefore appealing to (3.6) and to the preceding identity we have indeed established (3.8). Proceeding iteratively in a similar manner with the other components $\left(w_{2}^{1}, w_{3}^{1}, \ldots\right)$ we have indeed obtained $(3.7)$ and thus the proof of the lemma is complete.

\section{Existence of solutions}

We discuss the proofs of the two main theorems of the Introduction.

\subsection{The case of the determinant}

We recall Theorem 1.1.

Theorem 1.1 Let $\Omega \subset \mathbb{R}^{n}$ be a bounded open set, $\alpha<\beta$ and $0<\gamma_{2} \leq \ldots \leq \gamma_{n}$ be such that

$$
\gamma_{2} \prod_{i=2}^{n} \gamma_{i}>\max \{|\alpha|,|\beta|\} .
$$

Let $\varphi \in C_{\text {piec }}^{1}\left(\bar{\Omega} ; \mathbb{R}^{n}\right)$ (the set of piecewise $C^{1}$ maps) be such that, for almost every $x \in \Omega$,

$$
\left\{\begin{array}{l}
\alpha<\operatorname{det} D \varphi(x)<\beta, \\
\prod_{i=\nu}^{n} \lambda_{i}(D \varphi(x))<\prod_{i=\nu}^{n} \gamma_{i}, \nu=2, \ldots, n,
\end{array}\right.
$$


then there exists $u \in \varphi+W_{0}^{1, \infty}\left(\Omega ; \mathbb{R}^{n}\right)$ so that

$$
\left\{\begin{array}{l}
\operatorname{det} D u \in\{\alpha, \beta\}, \text { a.e. in } \Omega, \\
\lambda_{\nu}(D u)=\gamma_{\nu}, \nu=2, \ldots, n, \text { a.e. in } \Omega .
\end{array}\right.
$$

Proof. We now show that the result follows from the combination of Theorem 2.3 and Theorem 3.1. From Theorem 3.1 we have

$$
\begin{gathered}
E=\left\{\xi \in \mathbb{R}^{n \times n}: \operatorname{det} \xi \in\{\alpha, \beta\}, \lambda_{i}(\xi)=\gamma_{i}, i=2, \ldots, n\right\}, \\
\operatorname{Rco} E=\left\{\xi \in \mathbb{R}^{n \times n}: \operatorname{det} \xi \in[\alpha, \beta], \prod_{i=\nu}^{n} \lambda_{i}(\xi) \leq \prod_{i=\nu}^{n} \gamma_{i}, \nu=2, \ldots, n\right\} .
\end{gathered}
$$

Since $\varphi \in C_{\text {piec }}^{1}\left(\bar{\Omega} ; \mathbb{R}^{n}\right)$ and $D \varphi \in \operatorname{int}$ Rco $E$ we only need to verify that $E$ and Rco $E$ have the approximation property.

For $\delta>0$ such that $\gamma_{2}-\delta>0$ and $\alpha+\delta<\beta-\delta$, let

$$
E_{\delta}=\left\{\xi \in \mathbb{R}^{n \times n}: \operatorname{det} \xi \in\{\alpha+\delta, \beta-\delta\}, \lambda_{i}(\xi)=\gamma_{i}-\delta, i=2, \ldots, n\right\} .
$$

For a sufficiently small $\delta$ we have

$$
\left(\gamma_{2}-\delta\right) \prod_{i=2}^{n}\left(\gamma_{i}-\delta\right) \geq \max \{|\alpha+\delta|,|\beta-\delta|\}
$$

and thus Theorem 3.1 ensures that

$$
\operatorname{Rco} E_{\delta}=\left\{\begin{array}{c}
\operatorname{det} \xi \in[\alpha+\delta, \beta-\delta], \\
\xi \in \mathbb{R}^{n \times n}: \prod_{i=\nu}^{n} \lambda_{i}(\xi) \leq \prod_{i=\nu}^{n}\left(\gamma_{i}-\delta\right), \nu=2, \ldots, n
\end{array}\right\} .
$$

We have to verify the three conditions of Definition 2.2. The first one is obvious. We next verify the second condition. Since $\eta \in E_{\delta}$, we assume that $\operatorname{det} \eta=\alpha+\delta$, the case $\operatorname{det} \eta=\beta-\delta$ being handled in an analogous way. The set $E_{\delta}$ being left and right $S O(n)$ invariant, we can assume that

$$
\eta=\operatorname{diag}\left(\frac{\alpha+\delta}{\left(\gamma_{2}-\delta\right) \cdots\left(\gamma_{n}-\delta\right)}, \gamma_{2}-\delta, \ldots, \gamma_{n}-\delta\right)
$$

If we let

$$
\xi=\operatorname{diag}\left(\frac{\alpha}{\gamma_{2} \cdots \gamma_{n}}, \gamma_{2}, \ldots, \gamma_{n}\right)
$$

we have $\xi \in E$ and

$$
\operatorname{dist}(\eta ; E) \leq \max \left\{\left|\frac{\alpha+\delta}{\left(\gamma_{2}-\delta\right) \cdots\left(\gamma_{n}-\delta\right)}-\frac{\alpha}{\gamma_{2} \cdots \gamma_{n}}\right|, \delta\right\} \rightarrow 0, \text { as } \delta \rightarrow 0 .
$$

The second condition of Definition 2.2 then follows.

The third condition of the approximation property follows from the continuity of the functions involved in the definition of Rco $E_{\delta}$. We may then apply Theorem 2.3 to get the result. 


\subsection{The case of a quasiaffine function}

We recall Theorem 1.3.

Theorem 1.3 Let $\Omega \subset \mathbb{R}^{n}$ be a bounded open set, $\alpha<\beta, \Phi: \mathbb{R}^{m \times n} \longrightarrow \mathbb{R} a$ non constant quasiaffine function and $\varphi \in C_{\text {piec }}^{1}\left(\bar{\Omega} ; \mathbb{R}^{m}\right)$ such that, for almost every $x \in \Omega$,

$$
\alpha<\Phi(D \varphi(x))<\beta .
$$

Then there exists $u \in \varphi+W_{0}^{1, \infty}\left(\Omega ; \mathbb{R}^{m}\right)$ satisfying

$$
\Phi(D u) \in\{\alpha, \beta\} \text {, a.e. in } \Omega \text {. }
$$

Remark 4.1 The theorem is in fact slightly more precise and asserts also that if $c_{j}^{i}, i=1, \ldots, m, j=1, \ldots, n$ are constants such that $\left|D_{j} \varphi^{i}(x)\right|<c_{j}^{i}$ and

$$
|\Phi(\xi)|>\max \{|\alpha|,|\beta|\}, \forall \xi \in \mathbb{R}^{m \times n},\left|\xi_{j}^{i}\right|=c_{j}^{i}, i=1, \ldots, m, j=1, \ldots, n
$$

then the solutions also verify

$$
\left|D_{j} u^{i}(x)\right| \leq c_{j}^{i}, \forall(i, j) .
$$

Proof. As $\varphi \in C_{\text {piec }}^{1}\left(\bar{\Omega} ; \mathbb{R}^{m}\right)$, by Lemma 3.3, we can find constants $c_{j}^{i}$ such that $\left|D_{j} \varphi^{i}(x)\right|<c_{j}^{i}$ and

$$
|\Phi(\xi)|>\max \{|\alpha|,|\beta|\}, \forall \xi \in \mathbb{R}^{m \times n},\left|\xi_{j}^{i}\right|=c_{j}^{i}, i=1, \ldots, m, j=1, \ldots, n .
$$

We then define

$$
E=\left\{\xi \in \mathbb{R}^{m \times n}: \Phi(\xi) \in\{\alpha, \beta\},\left|\xi_{j}^{i}\right| \leq c_{j}^{i}, i=1, \ldots, m, j=1, \ldots, n\right\} .
$$

As before we only need to verify that the sets $E$ and Rco $E$ have the approximation property.

Let

$$
E_{\delta}=\left\{\xi \in \mathbb{R}^{m \times n}: \begin{array}{l}
\Phi(\xi) \in\{\alpha+\delta, \beta-\delta\} \\
\left|\xi_{j}^{i}\right| \leq c_{j}^{i}-\delta, i=1, \ldots, m, j=1, \ldots, n
\end{array}\right\}
$$

We first observe that, by continuity, it follows from (4.1) that

$$
|\Phi(\xi)|>\max \{|\alpha+\delta|,|\beta-\delta|\}, \forall \xi \in \mathbb{R}^{m \times n},\left|\xi_{j}^{i}\right|=c_{j}^{i}-\delta, \forall(i, j) .
$$

We can then apply Theorem 3.5 to find

$$
\operatorname{Rco} E_{\delta}=\left\{\xi \in \mathbb{R}^{m \times n}: \begin{array}{l}
\Phi(\xi) \in[\alpha+\delta, \beta-\delta] \\
\left|\xi_{j}^{i}\right| \leq c_{j}^{i}-\delta, i=1, \ldots, m, j=1, \ldots, n
\end{array}\right\} .
$$

It imediately follows that the first and third conditions of Definition 2.2 are verified. It therefore remains to check the second one.

We proceed by contradiction and assume that there exist $\varepsilon>0$ and a sequence $\eta_{n} \in E_{1 / n}$ with $\operatorname{dist}\left(\eta_{n}, E\right)>\varepsilon$. As $\left|\left(\eta_{n}\right)_{j}^{i}\right| \leq c_{j}^{i}$ we can extract a convergent subsequence, still denoted $\eta_{n}$, and $\eta \in E$ so that $\eta_{n} \rightarrow \eta$, which is at odd with $\operatorname{dist}\left(\eta_{n}, E\right)>\varepsilon$.

We can therefore invoke Theorem 2.3 to conclude the proof. 


\section{Existence of minimizers}

We consider in this section the minimization problem,

$$
\inf \left\{\int_{\Omega} g(\Phi(D u(x))) d x: u \in \varphi+W_{0}^{1, \infty}\left(\Omega ; \mathbb{R}^{m}\right)\right\}
$$

where $\Omega$ is a bounded open set of $\mathbb{R}^{n}, \varphi \in W^{1, \infty}\left(\Omega ; \mathbb{R}^{m}\right)$ and

$-g: \mathbb{R} \longrightarrow \overline{\mathbb{R}}=\mathbb{R} \cup\{+\infty\}$ is a lower-semicontinuous non convex function,

$-\Phi: \mathbb{R}^{m \times n} \longrightarrow \mathbb{R}$ is quasiaffine and non constant.

We recall that in particular we can have, when $m=n, \Phi(\xi)=\operatorname{det} \xi$.

The existence result for the problem $(P)$ that we will give is based on the assumption that the relaxed problem

$$
(Q P) \quad \inf \left\{\int_{\Omega} C g(\Phi(D u(x))) d x: u \in \varphi+W_{0}^{1, \infty}\left(\Omega ; \mathbb{R}^{m}\right)\right\},
$$

where $C g$ is the convex envelope of $g$, has piecewise $C^{1}$ solutions. If $\varphi$ is affine this is trivial, since $\bar{u}=\varphi$ is then a solution of $(Q P)$. When $\varphi$ is not affine the only result available is [6] valid for $m=n$ and $\Phi(\xi)=\operatorname{det} \xi$.

The existence result is the following.

Theorem 5.1 Let $\Omega \subset \mathbb{R}^{n}$ be a bounded open set, $g: \mathbb{R} \longrightarrow \overline{\mathbb{R}}=\mathbb{R} \cup\{+\infty\}$ a lower-semicontinuous function such that

$$
\lim _{|t| \rightarrow+\infty} \frac{g(t)}{|t|}=+\infty
$$

and $\varphi \in W^{1, \infty}\left(\Omega ; \mathbb{R}^{m}\right)$. If $(Q P)$ has a solution $u_{0} \in C_{\text {piec }}^{1}\left(\bar{\Omega} ; \mathbb{R}^{m}\right)$ then there exists $\bar{u} \in \varphi+W_{0}^{1, \infty}\left(\Omega ; \mathbb{R}^{m}\right)$ solution of $(P)$.

Proof. Let

$$
K=\{t \in \mathbb{R}: C g(t)<g(t)\} .
$$

The assumptions on $g$ ensure that $K$ is open and that it can be written as a countable union of disjoint bounded intervals:

$$
K=\bigcup_{j \in \mathbb{N}}\left(\alpha_{j}, \beta_{j}\right) .
$$

Moreover on every $\left[\alpha_{j}, \beta_{j}\right]$ the function $C g$ is affine i.e.

$$
C g(t)=a_{j}+b_{j} t, t \in\left[\alpha_{j}, \beta_{j}\right] .
$$

We then let

$$
\begin{aligned}
& \Omega_{0}=\left\{x \in \Omega: g\left(\Phi\left(D u_{0}(x)\right)\right)=C g\left(\Phi\left(D u_{0}(x)\right)\right)\right\} \\
& \Omega_{j}=\left\{x \in \Omega: \Phi\left(D u_{0}(x)\right) \in\left(\alpha_{j}, \beta_{j}\right)\right\}, j=1,2, \ldots
\end{aligned}
$$


Since $u_{0}$ is piecewise $C^{1}$, we find that the sets $\Omega_{j}, j=1,2, \ldots$ are open.

For every $j=1,2, \ldots$ so that $\Omega_{j} \neq \emptyset$, we apply Theorem 1.3 , with $\varphi=u_{0} \in$ $C_{p i e c}^{1}\left(\bar{\Omega}_{j} ; \mathbb{R}^{m}\right)$. We obtain in that way the existence of $u_{j} \in u_{0}+W_{0}^{1, \infty}\left(\Omega_{j} ; \mathbb{R}^{m}\right)$ so that

$$
\Phi\left(D u_{j}\right) \in\left\{\alpha_{j}, \beta_{j}\right\} \text {, a.e. in } \Omega_{j} .
$$

If we define

$$
\bar{u}= \begin{cases}u_{0}, & \text { in } \Omega_{0} \\ u_{j}, & \text { in } \Omega_{j}, j \in \mathbb{N},\end{cases}
$$

we have

$$
g(\Phi(D \bar{u}))=C g(\Phi(D \bar{u})), \text { a.e. in } \Omega .
$$

We claim that $\bar{u}$ is a solution of $(P)$. Indeed we have $\bar{u} \in \varphi+W_{0}^{1, \infty}\left(\Omega ; \mathbb{R}^{m}\right)$. Moreover appealing to (5.2), (5.3) and Proposition 2.5 we obtain

$$
\begin{aligned}
\int_{\Omega} g(\Phi(D \bar{u}(x))) d x & =\int_{\Omega} C g(\Phi(D \bar{u}(x))) d x \\
& =\sum_{j=0}^{\infty} \int_{\Omega_{j}} C g\left(\Phi\left(D u_{j}(x)\right)\right) d x \\
& =\int_{\Omega_{0}} C g\left(\Phi\left(D u_{0}(x)\right)\right) d x+\sum_{j=1}^{\infty} \int_{\Omega_{j}}\left(a_{j}+b_{j} \Phi\left(D u_{j}(x)\right)\right) d x \\
& =\int_{\Omega_{0}} C g\left(\Phi\left(D u_{0}(x)\right)\right) d x+\sum_{j=1}^{\infty} \int_{\Omega_{j}}\left(a_{j}+b_{j} \Phi\left(D u_{0}(x)\right)\right) d x \\
& =\int_{\Omega} C g\left(\Phi\left(D u_{0}(x)\right)\right) d x .
\end{aligned}
$$

Finally, using the fact that $u_{0}$ is a solution of $(Q P)$ and $\inf (Q P) \leq \inf (P)$, we obtain that $\bar{u}$ is a solution of $(P)$.

Acknowledgement: The research of A. M. Ribeiro was partially supported by portuguese Fundação para a Ciência e Tecnologia (BD/10042/02). Besides A. M. Ribeiro is on leave from FCT-UNL, Lisbon.

\section{References}

[1] A. Cellina and S. Zagatti. An existence result in a problem of the vectorial case of the calculus of variations. SIAM J. Control Optim., 33(3):960-970, 1995.

[2] B. Dacorogna. A relaxation theorem and its application to the equilibrium of gases. Arch. Rational Mech. Anal., 77(4):359-386, 1981.

[3] B. Dacorogna. Direct methods in the calculus of variations, volume 78 of Applied Mathematical Sciences. Springer-Verlag, Berlin, 1989. 
[4] B. Dacorogna and P. Marcellini. Existence of minimizers for nonquasiconvex integrals. Arch. Rational Mech. Anal., 131(4):359-399, 1995.

[5] B. Dacorogna and P. Marcellini. Implicit partial differential equations. Progress in Nonlinear Differential Equations and their Applications, 37. Birkhäuser, Boston, 1999.

[6] B. Dacorogna and J. Moser. On a partial differential equation involving the Jacobian determinant. Ann. Inst. H. Poincaré Anal. Non Linéaire, $7(1): 1-26,1990$.

[7] B. Dacorogna and G. Pisante. A general existence theorem for differential inclusions in the vector valued case. to appear.

[8] B. Dacorogna and C. Tanteri. On the different convex hulls of sets involving singular values. Proc. Roy. Soc. Edinburgh Sect. A, 128(6):1261-1280, 1998.

[9] B. Dacorogna and C. Tanteri. Implicit partial differential equations and the constraints of nonlinear elasticity. J. Math. Pures Appl., 81(4):311-341, 2002 .

[10] E. Mascolo and R. Schianchi. Existence theorems for nonconvex problems. J. Math. Pures Appl., 62(3):349-359, 1983.

[11] A. M. Ribeiro. Ph.D thesis (EPFL). 